\title{
Toward Pragmatic and Integrated Health Policies Reforms and Transformation in Africa
}

\author{
Tambo $\mathrm{E}^{1,2 *}$ \\ 1Department Biochemistry and Pharmaceutical Sciences, Université des Montagnes, \\ Cameroon \\ ${ }^{2}$ Africa Disease Intelligence and Surveillance, Communication and Response (Africa \\ DISCoR) Institute, Cameroon
}

\section{Review Article \\ Volume 1 Issue 1}

Received Date: November 13, 2017

Published Date: December 06, 2017

*Corresponding author: Ernest Tambo, Biochemistry and Pharmaceutical Sciences Department, Higher Institute of Health Sciences, Université des Montagnes, Cameroon; Africa Intelligence and Surveillance, Communication and Response (Africa DISCoR) Foundation, Yaoundé, Cameroon, E-mail: tambo0711@gmail.com

\section{Abstract}

The significant economic imperatives and ramifications of rising emerging chronic disease epidemics unmet health needs and persisting infectious diseases public health burden is huge across Africa. The article assesses unmet chronic diseases threats and epidemics need and potential threats and emerging issues in strengthening and deepening pragmatic health policy reforms and contextual transformations needed. We call for immediate collective partnership and action to implement innovative and pragmatic initiatives to various facets of personalized telemedicine and artificial intelligence for health to community-centered health systems policy reforms and transformations. Strengthening inter-sectoral partnerships and collaboration is crucial in creating value-needs investment and new perspectives in improving quality preparedness, prevention and smart response needed in achieving the Universal Health Coverage (UHC) and Sustainable Development Goals (SDGs). Research collaboration and transformative solutions on preparedness, smart health system preventive and management solutions is critical needed multifaceted risk factors and emerging epidemics-related impacts in Africa. Hence, leveraging on telemedicine and artificial intelligence innovative and smart healthcare solutions are highlighted in tackling emerging and rising non-communicable disease epidemics, infectious diseases threats/epidemics related child-maternal mortality to health system drawbacks and ageing population dilemma. Also, the need to advance in Africa traditional medicine and digital health communication approaches integration and practice in promoting health information for all in remote Africa communities for national and global health security, ecological development and economic prosperity.

Keywords: Non-communicable diseases; Epidemics; Preparedness; Health systems; Health informatics 
Abbreviations: NCDs: Non-Communicable Diseases; SDGs: Sustainable Development Goals; UHC: Universal Health Coverage; WHO: World Health Organisation; IHR: International Health Regulations; CASTEP: China-Africa Science and Technology Partnership

\section{Introduction}

Africa health systems unmet needs and rising noncommunicable diseases (NCDs) pose a major challenge to all. About two out of every three deaths globally are attributed to NCDs and 80 per cent of these fatalities occur in low- and middle-income countries [1]. Most African countries continue to face the greatest burden of persisting infectious diseases emerging and resurgence threats and NCDs epidemics, weakening health systems and worsening most vulnerable populations health and economy. The vicious cycle of ill-health and poverty imperatives call for instant smart actions from governments, the private sector and multilateral agencies to implement pragmatic multifaceted populationscentered health systems initiatives. Strengthening good governance and the rule of law, accountability and transparency is needed in improving the quality of care delivery and populations well-being [1,2]. Scaling up for impact preparedness, alertness and prevention in health system upgrading, and inter-sectoral collaboration is essential in achieving the Africa Union vision 2063 agenda, UHC and SDGs.

Recognizing that health system is central to care and prerequisite for, an outcome of, and effective indicator that promotes equity and sustainable health systems [1]. The value of health capital in an economy dwarfs any other form of capital; that requires developing and sustaining robust leadership, policy reforms in strengthening health systems dynamic perspectives in Africa [2,3]. Moreover, strengthening innovative partnerships and collaborative approaches in health policy development and health industries, science and technology, and trade and economy have immense perspective to promote healthy lifestyle and populations' wellness. These transformations are needed in making substantial contributions in reshaping and advancing Africa healthier capabilities in line with Sustainable Development Goals (SDGs, 2030) and Africa Union Vision 2063 [2-4].

Contemporary Africa is increasingly facing growing health-unmet needs, related emerging threats and epidemic challenges with severe impact on health systems. Addressing these challenges and issues require innovative public-private partnerships including governments, the private sector and multilateral agencies, community engagement, participation and ownership of programs towards sustainable health, economy, society ecological and economic development [1-3]. To answer these potential health-related threats and epidemics impacts on economy, trade and food security require pragmatic and integrated health policy reforms, deepening research partnerships and collaboration in preparedness, prevention and mitigation on risk factors $[4,5]$. Moreover, developing and implementing smart surveillance and response indicators and metrics in the management of emerging and rising non-communicable disease epidemics coupled with persisting infectious diseases public health burden mainly child-maternal health mortality and ageing population illnesses. Moving forward requires collaborative and smart health systems policy reforms and digital innovative transformations integration (e.g., telemedicine, artificial health intelligence) in primary healthcare delivery systems in addressing national health needs, existing challenges and drawbacks. But also, in promoting cultural and healthy democratic values, quality population health Alma-Atta and rights and tenets towards shared social cohesion, growth and global economic prosperity.

The paper assesses unmet chronic diseases threats and epidemics need and potential threats and emerging issues in strengthening and deepening pragmatic health policy reforms and contextual transformations much needed.

\section{Strengthening Early Detection, Prevention and Management of Non-communicable Diseases Epidemics}

There is an urgent need to develop and implement a contextual holistic, multi-sectoral and life-course approaches and strategies in preparedness, prevention and effective management of emerging chronic diseases threats and infectious diseases epidemics risk mitigation and wide spread smart operational strategies and measures(e.g., detection, diagnosis, analysis, visualization, treatment). Leveraging on the penetration and integration of smart mobile health and digital technology and tools such as smart phones and wearable sensors is crucial in revamping heath systems capabilities and quality outcomes in rural and hard to reach populations is essential in early detection and prognostics, alertness in evolving risk factors and determinants (stress and anxiety, alcoholism and tobacco consumption, malnutrition, obesity epidemics). Leveraging on advances in telemedicine and artificial intelligence for health through building daily primary healthcare routine cycle and workflow management could be vital in new 
paradigm of smart and high sensitivity computer-aided detection, diagnostic accuracy and treatment decisionmaking amongst health professionals and care providers data sharing. These visual assisted deductive and inductive processes are needed in advancing learning and optimal use of evidence and knowledge gains in tackling the growing NCDs burden including cardiovascular disease, cancer, diabetes, kidney and respiratory diseases mortality as well as mental illnesses disability, genderbased violence and injuries related deaths in the region [4]. Promoting of healthy lifestyles and physical fitness through the development of healthy cities is also important to ensure a greater focus on prevention and fitness rather than reactive response or treatment to chronic conditions. Hence, evidence-based smart solutions through deep learning data process and quality care optimization is necessary for workforce efficiency and time benefits and building of holistic diagnostic and effective treatment algorithms model and predictions to support dual education, therapeutics plans and resources allocation or government spending. These are essential in adapting positive behavioural changes, long term lifestyle adaptations and choices on modifiable chronic diseases risk factors (e.g.: smoking cessation policies, nutrition/dietary behavioural changes, physical activity promotion, and counteraction against substance abuse) and averting preventable premature disability and deaths. Moreover, addressing societal, ecological, environmental and occupational health related risk factors (e.g.: deforestation, illicit mining consequences, gas emissions and pollution) is needed in tackling global warming and climate change, road traffic accidents and occupational workplace risks [2,3,5]. Early warning alert and surveillance systems of growing NCDs risky factors in promoting health information for all vulnerable and remote populations is needed for prompt and effective disability mitigation and poverty alleviation to quality care delivery for health and economic gains across Africa.

\section{Operationalizing New Paradigm of Health Transformation Programs Engagement and Investment}

Fostering evidence-based personalized telemedicine and health systems centered on community health services should improve services delivery, quality interventions and outcomes in cubing NCD risks and impact across Africa [6]. Striving to ensure clean water, sanitation and hygiene, increasing food production and nutrition capabilities such as mechanization underscores the negative impact on health and disease costs and benefits. Investing on sustainable population based basic immunization programs is critical in scaling up access and uptake coverage against vaccine preventable diseases, nurturing continuous consumption of new and underused vaccines in emerging or resurgence of pandemics threats or epidemics consequences [1,3,5]. Developing countries governments and stakeholders' efforts should also be directed to intensifying community awareness, social mobilization and participatory engagement in knowledge and risk-dissemination, sexual and reproductive health in achieving universal health coverage and sustainable development goals (SDGs) agenda.

For example, promoting "Every Woman Every Child" initiative to eliminate preventable child and maternal morbidity and death relies on ensuring universal access to and scale coverage of childhood and maternal immunization packages and high quality diagnostic and treatment against chronic diseases including persisting HIV/AIDS, tuberculosis and malaria co-infections $[1,6]$. As, the rising NCDs epidemics continue to impose additional healthcare unmet needs, new paradigm of health transformation perspective should be built on collective engagement and investment for timely advocacy and risk mitigation against potential public health emergencies $[1,2,6]$. Likewise, monitoring and surveillance of drugs resistance is needed in fostering ethics and adherence of patient-health and professionalcare provider collaboration and cooperative learning in optimizing care quality and outcomes. Building robust and sustainable capacity building is also needed in increasing population health literacy, knowledge and resilience of threats and diseases, protected health information policy and regulatory systems (e.g., data access, privacy, rights, confidentiality, data sharing) on medicine prescriptions and pharmaceutical products quality control. Fostering private-public partnership investment on safe and effective vaccines in scaling up local populations accessibility, availability and uptake of immunization programs, while strengthening local procurement and supply chain management are vital for healthcare commodities needs sustainability.

\section{Reinforcing Primary Healthcare Systems in UHC and SDGs Agenda Integration for Healthier Africa}

Guaranteeing long term sustainability mechanism and share experiences amongst stakeholders can provide the whole population including the poor, vulnerable and marginalized groups access to safe, effective and affordable primary healthcare in line with Universal Health Coverage and Abuja and Harare declarations, without the risk of suffering from financial hardship $[1,2,7]$. The establishment financially sustainable health 
systems with appropriately-trained workforce; with effective, interoperable health information systems can boost access to safe and effective essential medicines and vaccines $[7,8]$. Hence, improving the quality of medical services of primary health care institutions by improving the level of medical knowledge of primary health care providers, strengthening incentives for referral, and strengthening the integration of different levels of health system, while integrating traditional and complementary/alternative medicines into primary healthcare delivery. This should support cultural and traditional perceptions and practices motivations and contextual structures, while promoting governments and related stakeholders investment in attaining a minimum of $15 \%$ of the national annual budget health in accordance to Abuja Declaration, Pan Africanism and Africa Union Vision 2063 in advancing healthier future for Africa [6-9].

\section{Improving Health Early Warning and Surveillance Strategies in Emergency Preparedness and Response System}

Public health events and disasters emergency responses and applications of effective interventions in the region still continue to face several drawbacks and issues that need to be urgently prioritized by governments. Improving investment in real time, effective and contextual local, national and regional early digitalized warning and surveillance strategies are crucial in early detection and confirmation, preparedness and health risk mitigation tactics through effective coordination and monitoring systems in line with the World Health Organization and International Health Regulations (IHR, 2005) implementation $[6,7,10]$.

Evidence-based appropriate and sustainable resources allocation and expenditure is needed in strengthening robust and practical early detection of warning signals and surveillance of risk factors indicators in generating data for evidence-based policy decisions and smart response choices are needed. Timely monitoring and evaluation of interactive early signals and surveillance indices community on community behavioural and resilience is appropriate [7,9]. Real time community response simulations of public health emergencies are indispensable especially emerging and re-emerging infectious diseases outbreaks and disasters, and NCDs pandemic at all levels in Africa sub-region [1,3,10]. As well genuine efforts in strengthening inter sectoral and multi-disciplinary approaches in capacity building in health emergency preparedness and response workforce are needed. Also, promoting local to regional policy reforms and sustained leadership commitment in ensuring access and low-cost care services and upgrading basic primary health infrastructures capabilities of communities are crucial increasing fast coverage rate of rapid response and recovery from public health emergencies disasters.

\section{Fostering Community-based Health Approaches, Policies and Practice}

Fostering contextual community-based multi-sectoral and cross-borders programs and projects towards real time preparedness and smart response actions are critical. Evidence-based local, national and regional health policies development and transformative reforms implementation is crucial. Appropriate community monitoring and evaluation of policies and programs uptake, utilization and effectiveness is imperative in improving shared value and wellness. Proactive and resilient support from non-health sectors such as science and technology, commerce, agriculture, and environment and transportation implications should be stimulated to advance primary and homecare delivery towards attaining the UCH and shared health equity benefits $[7,8]$. Hence, nurturing sustained capacity building and empowerment at individuals and community level in enhancing and facilitating welfare and wellbeing (including marginalized and vulnerable groups such as children, women, disabled and migrant/refugee populations). Promoting community participatory approach towards programs and projects ownerships is vital in community health planning, enhanced legislation, services delivery, education, training, monitoring and knowledge dissemination advancements at all levels $[1,6]$.

Understanding existing challenges and conducting further research on socioeconomic, environmental and genetic determinants and risk factors on health and related consequences are imperative. Appropriate evidence-oriented in integrated decision policy and actions can reduce health inequities; creating an inclusive, productive and healthier society. Emboldening publicprivate partnership in strengthening collaborative leadership and financing accountability and transparency is critical to ease regulation and guidance at micro- and macro levels, necessary in boosting private sectors interest in health care industry, provision of public health goods and services $[2,3,7,9]$.

\section{The Value of Policy Reforms in NCDs Research Translation into Practice Transformation}

Promoting cooperation in added-value to health systems mechanisms in bio-medical sciences, health and health policy research and health development to

Tambo E. Toward Pragmatic and Integrated Health Policies Reforms and Transformation in Africa. J Inf Dis Trav Med 2017, 1(1): 000103. 
sustainable economic growth is imperative. Evidencebased strengthening basic and operational research towards evidence-based medical and health policy reforms, and cost-effective preventive and management interventions are needed to meet the health needs in the region $[10,11]$. Such national and regional low-cost and smart programs and interventions implementation are vital in increasing digital community health learning and educational outreach as well as awareness, promoting empowerment and alertness against harmful risk and barriers to quality healthy lives $[7,12]$. The value of policy reforms and regulations reinforcement such as salt, sugar and trans-saturated fats consumption or importation, tobacco and alcohol consumption burden underscores the urgent actions.

Evidence-based policy and open access information value in improving early detection, population-based awareness, empowerment and prompt resilience culture against NCDs risk and burden including obesity and related cardio metabolic diseases is essential. Publicprivate sectors investment in advancing research and development (R\&D) in WHO-AFRO members' innovative health education and promotion is imperative. Fostering sharing value and innovations transfer and knowledge exchanges should be of great value in grooming entrepreneurship culture and creativity in increasing capacities innovative knowledge practice and new opportunities in the region $[7,11,13]$.

The need to support local, regional and global initiatives in addressing health-related challenges through South-South or South-North partnership, while leveraging on full access and use of regional resources for economy growth and wellbeing. In addition, promoting regional trade and tarns-health capacity development can support economy development. Addressing current health commodities value chain is needed in identifying barriers in the supply chain that drive up costs and inhibit access to medical products by patients $[3,7,8,10,14]$. Meanwhile, nurturing shared health reform experiences and best practice collaboration can support good use of our resources. Strengthening regional own health systems and capacities and opportunities in disease prevention and control is capital in ensuring timely basic medical services accessibility and availability at all levels $[7,9,11,15]$. Fostering and strengthening evidence-based expansion of dialogue and communication, cooperation and coordination between trans-disciplinary and intersectorial health government, industry and civil society needed. Furthermore, tackling regional and sub-regional health challenges and issues in ensuring healthier, sustainable environments and access to digital health insurance (e.g., tele-diagnosis, teleconsulting and digital monitoring of medication adherence, etc...) where people live, work, study and play for social justice and equity [3,7,15-17].

\section{Conclusion}

Moving forward healthier African populations' quality care delivery and health systems transformation beyond emerging infectious disease threat and epidemics requires increasing collaborative commitment and investment in R\&D technology and tools, preparedness and resilience strategies against the rising NCDs public health burden. The paper calls for more strategic and coordinated health priorities and programmatic implementation of emerging NCD threats and epidemics approaches and interventions immense opportunities tailored to specific local and country contexts and needs based on advances of personalized-telemedicine (digital patient-centered) and integrated health systems prevention and management in developing countries especially in Africa. Strengthening participatory engagement, responsibility and accountability is an imperative for all stakeholders at all levels. In such as, novel sensitive diagnostics, safer medicines for management and monitoring wearable biosensors and smart devices for remote rural tele-diagnosis, teleconsultation and counseling, improving population awareness and educational empowerment for equitable health for all, positive behavioural changes and lifestyle adaptation strategies. Advancing community-based and national health insurance packages implementation is vital in broadening effective and efficient care delivery interventions and quality outcomes. Innovative telemedicine and artificial intelligence models and predictions are needed in building visionary healthy African leadership and sustainable partnerships, cooperation and collaboration management and public health governance transformations for diseases free society and future generations.

\section{Competing interests}

Author declares that they have no conflict interests

\section{Author's contributions}

ET conceived and designed the study and strategy. ET wrote the primary draft of the paper and provided additional information. The author read and approved the final version of the manuscript. 


\section{Journal of Infectious Diseases \& Travel Medicine}

\section{Acknowledgments}

ET is a recipient of China-Africa Science and Technology Partnership (CASTEP) Postdoctoral Fellowship award, 2011 and Career Development of S\&T Career Development Program Award, 2013.

\section{References}

1. World health Organization (WHO) (2015) Noncommunicable diseases.

2. Lee JT, Hamid F, Pati S, Atun R, Millett C (2015) Impact of Non-communicable Disease Multimorbidity on Healthcare Utilisation and Out-OfPocket Expenditures in Middle-Income Countries: Cross Sectional Analysis. PLoS One 10(7): e0127199.

3. Tambo E, Madjou G, Wandabwa CK, Tekwu EN, Olalubi OA, et al. (2016) Can free open access resources strengthen knowledge-based emerging public health priorities, policies and programs in Africa? F1000 Res 5:853.

4. Warren AE, Wyss K, Shakarishvili G (2013) Global health initiative investments and health systems strengthening: a content analysis of global fund investments. Global Health (England) 9(1): 30.

5. Jamison DT, Summers LH, Alleyne G (2013) Global health 2035: A world converging within a generation. The Lancet 382(9908): 1898-1955.

6. Andreu-Perez J, Leff DR, Ip HM, Yang GZ (2015) From Wearable Sensors to Smart Implants--Toward Pervasive and Personalized Healthcare. IEEE Trans Biomed Eng 62(12): 2750-2762.

7. Fryatt R, Mills A, Nordstrom A (2010) Financing of health systems to achieve the health Millennium Development Goals in low income countries. Lancet 375(9712): 419-426.

8. Tambo E, Ugwu EC, Ngogang JY (2014) Need of surveillance response systems to combat Ebola outbreaks and other emerging infectious diseases in African countries. Infect Dis Poverty 3: 29.
9. Hirschhorn LR, Baynes C, Sherr K (2013) Approaches to ensuring and improving quality in the context ofhealthsystemstrengthening: a cross-site analysis ofthe five AfricanHealth Initiative Partnership programs. BMC Health Serv Res 13: 2 pS8.

10. Frenk J (2010) The global health system: strengthening national health systems as the next step for global progress. PLoS Med 7(1): e1000089.

11. Mutale W, Chintu N, Amoroso C (2013) Improving health information systems for decision making across five sub-Saharan African countries: Implementation strategies from the African Health Initiative. BMC Health Serv Res 13 Suppl 2: S9.

12. Kutz D, Shankar K, Connelly K (2013) Making sense of mobile- and web-based wellness information technology: cross-generational study. J Med Internet Res 15(5): e83.

13. Tambo E, Khater EI, Chen JH, Bergquist R, Zhou XN (2015) Nobel prize for the artemisinin and ivermectin discoveries: a great boost towards elimination of the global infectious diseases of poverty. Infect Dis Poverty 4: 58.

14. Shakarishvili G, Atun R, Berman P, Hsiao W, Burgess $C$, et al. (2010) Converging Health Systems Frameworks: Towards A Conceptsto Actions Roadmap for Health Strengthening in Low and Middle Income Countries. Global Health Governance 3(2):17.

15. Tambo E, Yah CS, Ugwu CE, Olalubi OA, Wurie I, et al. (2016) Fostering prevention and care delivery services capability in Human Immunodeficiency virus and Ebola outbreak symbiosis in African vulnerable populations. J Infect Dev Ctries 10(1): 1-12.

16. Wamala D, Katamba A, Dworak O (2011) Feasibility and diagnostic accuracy of Internet-based dynamic telepathology between Uganda and Germany. J Telemed Telecare 17(5): 222-225.

17. Erikson H, Salzmann-Erikson M (2016) Future Challenges of Robotics and Artificial Intelligence in Nursing: What Can We Learn from Monsters in Popular Culture? Perm J Summer 20(3). 